\title{
El efecto de las experiencias de práctica en el desarrollo del sentido de autoeficacia en la formación inicial de educadoras de párvulos
}

\author{
The effect of field-based experiences in the development of the sense \\ of self-efficiency during the education of pre-school teachers \\ O efeito das experiências de prática no desenvolvimento do sentido da \\ auto-eficácia na formaçao inicial de professores de educação infantil
}

\author{
M. Francisca del Río ${ }^{1}$, Claudia Lagos ${ }^{2}$,Horacio Walker $^{3}$ \\ ${ }^{1}$ Facultad de Educación. Universidad Diego Portales, Chile. Fono: 6768536. E-mail: francisca.delrio@mail.udp.cl \\ ${ }^{2}$ Facultad de Educación. Universidad Diego Portales, Chile. Fono: 6768502. E-mail: claudia.lagos@udp.cl \\ ${ }^{3}$ Facultad de Educación. Universidad Diego Portales, Chile. Fono: 6768501. E-mail: horacio.walker@udp.cl
}

\section{RESUMEN}

El presente estudio aplicó la escala de autoeficacia de Tschannen-Moran y Woolfolk Hoy (2001) a un grupo promedio de 69 alumnas de la carrera de Pedagogía en Educación Parvularia de la Universidad Diego Portales, con el fin de conocer la evolución del sentido de autoeficacia durante sus estudios. Se hipotetizó que la eficacia percibida se debiera fortalecer a medida que se contaba con una mayor experiencia práctica en las aulas. Los resultados muestran una fluctuación de la autoeficacia entre los semestres de formación, con un comportamiento disímil de los diversos factores de la eficacia evaluados. Se aventuran explicaciones a los resultados, abriendo la puerta a futuras intervenciones dirigidas a fortalecer el sentido de eficacia de los profesores en formación.

Palabras clave: autoeficacia, educación inicial pedagógica.

\begin{abstract}
The present study applied the Tschannen-Moran \& Woolfolk (2001) self-efficiency scale to a sample group of an average of 69 teacher candidates enrolled in a Pre-school Education Teacher Preparation Program at Universidad Diego Portales in Chile. The objective of the study was to analyze the development of the self-efficiency of teacher candidates during their education. We hypothesized that the sense of efficiency reported would become stronger as candidates increased their exposure and participation in field-based experiences throughout the program. The results show a fluctuation of the self-efficiency among the training semesters, with a dissimilar behavior of the different factors of the efficiency evaluated. Explanations might be given about the results, opening the door for future interventions aiming at strengthening the sense of efficiency of the teacher trainees.
\end{abstract}

Key words: self-efficiency, teacher preparation programs.

\section{RESUMO}

O presente estudo aplicou a escala de auto-eficácia de Tschannen-Moran e Woolfolk Hoy (2001) a um grupo de aproximadamente 69 alunas do curso de Pedagogia em Educação Infantil da Universidade Diego Portales, com o objetivo de analisar a evolução do sentido de auto-eficácia em diversos estágios do programa de formação. A hipótese testada é que o sentido de eficácia se fortalece na medida em que se ganha maior experiência prática na sala de aula. Os resultados mostram uma flutuação da auto-eficácia entre os semestres de formação com um comportamento dissimilar dos diversos fatores de eficácia analisados. As explicações dadas aos resultados obtidos abrem a porta para futuras intervenções dirigidas ao fortalecimento do sentido de eficácia de professores em formação.

Palabras-chaves: auto-eficácia, educação inicial pedagógica. 


\section{INTRODUCCION}

El sentido de autoeficacia de los profesores es un constructo simple, pero de consecuencias altamente significativas para la educación. Diversas investigaciones han demostrado que el sentido de autoeficacia juega un rol central en determinar la calidad de la labor docente de los profesores y, por lo tanto, tiene también un efecto directo en el rendimiento de sus alumnos (Milner, 2002; Shaughnessy, 2004; Tournaki \& Podell, 2005).

De esta manera, favorecer un sentido de autoeficacia positivo resulta una tarea central en los programas de formación inicial docente. Si bien la mayor parte de los programas de formación inicial pone especial acento en entregar una formación teórica sólida, las investigaciones han mostrado que justamente son las experiencias prácticas durante la formación las que más directamente promueven un juicio de autoeficacia positivo (Richards \& Clough, 2004).

En esta lógica, la carrera de Educación Parvularia de la Universidad Diego Portales (en adelante UDP) pone especial acento, durante el transcurso de la formación, en un contacto precoz e intensivo con la realidad educativa. Esto se traduce en que el eje de prácticas esté presente de manera transversal a lo largo de toda la formación. Así, de este tratamiento intensivo de contacto con la realidad educativa del aula podría esperarse como resultado un efecto positivo en el sentido de autoeficacia de los alumnos/as de la carrera. Esta presunción nos lleva al objetivo de la presente investigación, la que busca conocer la evolución del sentido de autoeficacia de los estudiantes de pedagogía en educación parvularia, hipotetizando que éste debiera fortalecerse a medida que los estudiantes avanzan en su formación y se ven inmersos en una mayor cantidad de experiencias prácticas de aula.

Para responder esta pregunta es que se aplicó a los estudiantes de cada semestre de la carrera de Educación Parvularia una escala de autoeficacia, con el fin de comparar su rendimiento de acuerdo al semestre de carrera que cursaban.

Se espera que los datos obtenidos por la presente investigación apoyen la revisión de los principales postulados de la apuesta de la Facultad de Educación de la Universidad Diego Portales respecto al eje de formación práctica. Por otra parte, es importante hacer notar que esta iniciativa se enmarca en un proyecto de mayor escala, un proyecto FONDECYT ( $n^{\circ}$ 1070807) que persigue conocer cómo se aprende a enseñar, iniciativa donde, entre otras medidas, se evalúa el nivel de autoeficacia de los estudiantes de pedagogía en educación básica y media de numerosas universidades.

\section{MARCO TEORICO}

\section{CONCEPTO DE AUTOEFICACIA}

La creencia de los profesores acerca de su eficacia es definida como un juicio respecto de su propia capacidad para lograr resultados positivos de aprendizaje entre sus alumnos, aun entre aquellos que puedan tener dificultades o estar poco motivados (Armor et al., 1976; Bandura, 1977; Woolfolk Hoy \& Burke Spero, 2005). O, en términos más concretos, el sentido de autoeficacia de los profesores se puede definir como las creencias individuales de un profesor acerca de sus capacidades para llevar a cabo ciertas tareas 
específicas de su labor, a un nivel específico y en una situación particular. También, en otras palabras, se refiere a la opinión o juicio manifestado por un profesor relativo a la propia capacidad percibida para incrementar tanto los resultados de aprendizaje como la implicación académica de los alumnos a los que instruye (Woolfolk Hoy \& Burke Spero, 2005).

De acuerdo a Bandura (1997), las creencias de autoeficacia conforman un factor dinámico de corte personal que resulta crucial para estudiar la disposición de las personas a actuar. Esto, pues las creencias de autoeficacia actuarían como mediadoras en la relación entre el conocimiento y el comportamiento. Así, debido a su carácter anticipatorio, las expectativas de autoeficacia determinarán la probabilidad del profesor de llevar a cabo ciertas acciones instrumentales, el esfuerzo que se invertirá y el grado de perseverancia de un comportamiento particular ante la aparición de obstáculos o dificultades. Pero, por otra parte, las creencias de autoeficacia no se consideran como un rasgo estable de un sujeto, sino como un sistema de creencias activo y en pleno intercambio con el medio (Bandura, 1997). De esta forma, estas creencias pueden ser sujeto de intervenciones, variando de acuerdo al contexto y a la tarea a la que se refieren.

Diversas investigaciones han dado cuenta de la relevancia que el sentido de autoeficacia de los profesores tiene en el ámbito educativo. Así, se ha mostrado que las creencias que los profesores sostienen acerca de sus propias capacidades para enfrentar los desafíos de su labor juegan un importante rol en el grado de compromiso con que enfrentan su trabajo (Skaalvik \& Skaalvik, 2007; Tschannen-Moran \& Woolfolk Hoy, 2001; Wolters \& Daugherty, 2007). De esta forma, se ha demostrado que el sentido de autoeficacia de los profesores se relaciona a su comportamiento en la sala de clases. La eficacia percibida afecta el monto de esfuerzo que invierten en enseñar, las metas que se fijan y su nivel de expectativas. Asimismo, los profesores con un alto sentido de autoeficacia tienen una mayor probabilidad de presentar mayores niveles de planificación y organización (Allinder, 1994; Milner, 2002). También son más abiertos a nuevas ideas y más dispuestos a experimentar con nuevos métodos con el fin de ofrecer más oportunidades a sus alumnos (Stein \& Wang, 1988). Por otra parte, las creencias de eficacia influencian la persistencia de los profesores antes situaciones difíciles, y los llevan a ser menos críticos con los estudiantes que cometen errores (Ashton \& Webb, 1986). Aún más, los profesores con un alto sentido de autoeficacia presentan la tendencia a ser más entusiastas al enseñar (Allinder, 1994) y una mayor probabilidad a mantenerse enseñando a lo largo del tiempo (Glickman \& Tamashiro, 1982).

Otra línea de investigación ha demostrado que las creencias de autoeficacia de los profesores son una importante cualidad docente que mantiene relación con el logro académico de sus alumnos, o en otras palabras, que pueden influenciar positivamente el aprendizaje de sus alumnos (Shaughnessy, 2004; Tournaki \& Podell, 2005). En efecto, el sentido de autoeficacia de los profesores se relaciona con mayores niveles de logro académico y de motivación en los alumnos (Goddard, Hoy \& Woolfolk Hoy, 2004). Por el contrario, los profesores con menores niveles de autoeficacia tienen mayores dificultades con el comportamiento de los estudiantes, son pesimistas acerca del aprendizaje de sus alumnos, y experimentan mayores de niveles de estrés y menores niveles de satisfacción laboral (Bandura, 1997; Caprara, Barbaranelli, Steca \& Malone, 2006).

Todos estos antecedentes dan cuenta de la importancia del sentido de autoeficacia para la labor de un profesor, lo que nos lleva a plantearnos la relevancia de abordar este 
tema durante la formación inicial pedagógica. Así, conocer el comportamiento de este constructo entre los estudiantes de pedagogía a lo largo de su formación -objetivo de esta investigación- constituye la oportunidad de contar con datos de gran valor pues, por un parte, representa el reflejo de la labor de enseñanza que una propuesta de formación de profesores lleva a cabo con sus alumnos y, por otra, abre la puerta a una intervención dirigida a proveer, durante esta formación, actividades y experiencias dirigidas a fortalecer el sentido de autoeficacia.

\section{EL EFECTO DE LA FORMACION PRACTICA EN EL SENTIDO DE AUTOEFICACIA}

Además de conocer la evolución del sentido de eficacia durante el desarrollo de los estudios universitarios de educación parvularia, esta investigación se propone analizar el papel que juegan las experiencias de práctica, desarrolladas por estudiantes de pedagogía en educación parvularia durante su formación inicial, en la configuración de sus creencias de autoeficacia. Al respecto, postulamos que una formación pedagógica que pone especial acento en promover la interacción de los estudiantes con los contextos reales de enseñanza debiera -al darles la oportunidad de poner en práctica los conocimientos y habilidades adquiridas- fortalecer el sentido de autoeficacia docente de los estudiantes.

Contrariamente, los programas de formación de profesores tienden a sobrevalorar la importancia de adquirir conocimiento teórico, en detrimento de equipar a los estudiantes con experiencias de campo comparables a las situaciones que enfrentarán como futuros docentes (Carroll, Forlin \& Jobling, 2003). Esto resulta contradictorio, pues tal como ya se ha mencionado, se ha determinado que el mayor beneficio que los alumnos pueden extraer de la formación docente es justamente el entrenamiento en terreno, experiencia en la que tendrán la oportunidad de contextualizar el aprendizaje adquirido (Richards \& Clough, 2004).

En efecto, actualmente existe un amplio consenso entre los expertos en educación en considerar el aprendizaje como una actividad situada (Putnam \& Borko, 2000), es decir, que debe ocurrir en el marco de experiencias reales, auténticas y cotidianas (Díaz \& Hernández, 1998). Este planteamiento, emanado desde la perspectiva de la cognición situada (Brown, Collins \& Duguid, 1989), considera que el aprendizaje de calidad se desarrolla en procesos y contextos transaccionales (Bruner, 1997) y que éste es, ante todo, una construcción de significados. De tal manera que quien aprende pasa a constituirse en un ente interpretativo de la realidad en que está inmerso y, necesariamente para llevar a cabo dicha construcción, debe interactuar, dialogar y negociar sus propios significados con el entorno en que interactúa (Molina, 2000). Esto ha llevado a privilegiar el que los estudiantes cuenten durante su formación inicial pedagógica con la oportunidad de tener experiencias de práctica en terreno, asumiendo que una temprana vinculación de los futuros profesores con el contexto en el que han de desempeñarse profesionalmente resultará beneficiosa para su formación.

Sin embargo, se ha observado que esta vinculación con el sistema escolar suele ser compleja y, en algunos casos, contradictoria (Clift \& Brady, 2005), entre otras razones debido a que dichos contextos no necesariamente ilustran las perspectivas actualizadas sobre enseñanza y aprendizaje que se desea promover en los programas de formación de profesores (Putnam \& Borko, 2000; Bullough, 2000). De este modo, estas experiencias no siempre estarían otorgando a los estudiantes de pedagogía genuinas oportunidades ni para aprender a enseñar, ni para desarrollar un sentido de autoeficacia positivo. 
En este sentido conviene preguntarse si, siendo el proceso de formación de un profesor un momento tan fuertemente condicionado por la interacción entre éste y el contexto en que se desenvuelve (Bullough, 2000), el estudiante logra, a partir de las experiencias de formación práctica que vivencia, resignificar sus creencias de autoeficacia.

En este marco, Pigge y Marso (1990) llevaron a cabo un estudio para determinar si es que la formación inicial docente presentaba un impacto positivo en las atribuciones emocionales de los futuros profesores. Medidas de ansiedad, actitudes, preocupaciones y sentido de autoeficacia para enseñar fueron administradas a una muestra de 153 profesores en formación, tanto al inicio de la formación como al completarla. Los hallazgos mostraron que a medida que avanzaban en sus estudios los profesores de preocupaban menos de la posibilidad de sobrevivir en esta labor y más por su performance en la labor de enseñar, sentían menor ansiedad respecto a enseñar, y se sentían más seguros de su decisión vocacional. Asimismo, se encontró que durante toda la formación se mantenía un alto nivel de sentido de eficacia respecto a ser profesores exitosos en el futuro, sus aptitudes para enseñar y para causar un buen impacto en sus futuros alumnos.

Por otra parte, un estudio realizado por Conway y Clark (2003) acerca de la formación práctica de los docentes en formación en la Universidad de Michigan mostró que las influencias organizacionales y contextuales en que se circunscriben estas experiencias resultan claves para la automejora y el desarrollo de un profesor en formación. Específicamente, en el caso de este programa -que promueve la formación de estudiantes con una postura reflexiva frente a la enseñanza- los alumnos experimentaron un patrón de desarrollo igualmente orientado hacia lo externo -enseñando contenidos y preocupándose por sus estudiantes-, como hacia lo interno, focalizándose en su supervivencia, identidad, automejora y autodesarrollo.

Así, es posible establecer que la condición situada del aprendizaje anteriormente señalada alude tanto a las características contextuales del espacio en que tiene lugar la experiencia de formación práctica en el sistema escolar, como a las oportunidades de reflexionar acerca de dicha experiencia que otorga el contexto formativo en el que ésta se enmarca. Esto, porque si bien la experiencia de práctica otorga el contenido sobre el cual reflexionar, el que ello ocurra dependerá de las competencias reflexivas individuales que posea el futuro profesor y de la efectividad de cómo éstas se intencionen durante la formación, por cuanto de lo que se trata es de poner en acción un razonamiento pedagógico que permita al estudiante enfrentarse a la complejidad del aula y la toma de decisiones desde una perspectiva pedagógica (Shulman, 2005). Sin embargo, la toma de conciencia que alcanza el estudiante, presumiblemente producto de la adquisición y desarrollo de un razonamiento pedagógico que le permitiría analizar la performance de su enseñanza desde otro cariz, podría eventualmente implicar el verse desprovisto de un sentido de autoeficacia positivo.

Al respecto, un estudio realizado en la Universidad Católica de Temuco (Del Valle et al., 2009) con estudiantes de la carrera de pedagogía en inglés reveló que las experiencias prácticas que desarrollan los estudiantes durante un trayecto formativo de cuatro años, más que contribuir a fortalecer la consolidación del sentido de autoeficacia entre los estudiantes, lo debilitaban. Ello podría explicarse probablemente a partir de la "toma de conciencia" antes señalada.

Dado que existe evidencia en la literatura que muestra que el sentido de autoeficacia de los profesores en formación puede aumentar a medida que el programa progresa, y otra que muestra lo contrario y, que asimismo, la autoeficacia puede también decrecer 
una vez que los estudiantes comienzan a enseñar en las escuelas (Hoy \& Woolfolk, 1993; Tschannen-Moran, Woolfolk Hoy \& Hoy, 1998), resulta crucial que los programas de formación docente provean de un espacio para el desarrollo de destrezas prácticas, porque son justamente este tipo de experiencias prácticas las que acompañadas de una reflexión guiada tendrán un impacto positivo en el sentido de autoeficacia de los profesores en formación. Así, no queda duda de que las experiencias de práctica son importantes para los profesores en formación, pues son esas experiencias las que les entregan el recurso más relevante de la autoeficacia docente, la sensación de logro en su performance, la que proviene justamente de la interacción y experiencia de trabajar con alumnos y enseñar en aulas reales.

\section{PROPUESTA FORMATIVA UDP}

En la realidad particular de la carrera de Educación Parvularia de la UDP, las experiencias de formación práctica que desarrollan los estudiantes durante los ocho semestres que esta contempla asumen la relevancia del aprendizaje situado que propicia la construcción personal y colectiva de significados desde las dos perspectivas teóricas anteriormente señaladas.

De este modo, las actividades prácticas tienen lugar en espacios y contextos sociales diversos: la universidad, el sistema escolar, el aula, e involucran a distintos actores: estudiantes, académicos, mentores, entre otros, propiciando en el profesor en formación una reflexión sobre la experiencia, que le permita identificar y resignificar sus supuestos preprofesionales sobre la enseñanza y el aprendizaje y los contextos en que éstos se desarrollan.

Por ello, se debe velar por la articulación de estas distintas entidades, asumiendo su complejidad, estableciendo relaciones entre todas ellas y propiciando la construcción, por parte de los futuros educadores, de los diversos saberes que están a la base del ejercicio de la docencia, brindándoles oportunidades que les posibiliten aprender a enseñar.

\section{Figura 1}

El modelo teórico que se presenta a continuación refleja lo recién expuesto:

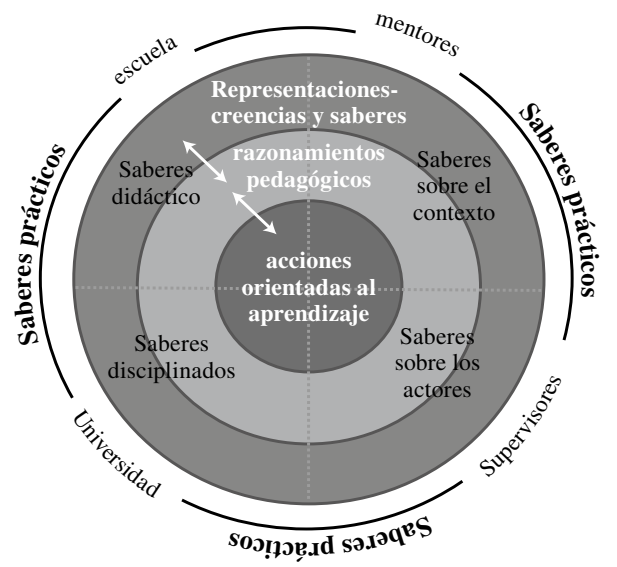

(Lagos, Meneses y Walker, 2008). 
Así, este modelo pretende que durante el trayecto formativo los estudiantes puedan acceder a experiencias en la amplia variedad de contextos educativos que ofrece el sistema escolar chileno, incorporando paralelamente a cada experiencia un taller de práctica que intenta acompañar la experiencia y guiar la reflexión de los estudiantes. De este modo, la experiencia se organiza de la siguiente manera:

\section{Tabla 1}

Caracterización de los talleres de práctica en la carrera de Pedagogía en Educación Parvularia de la Facultad Educación UDP a partir del análisis de sus programas de estudio y las experiencias que desarrollan en el terreno, año 2008

\begin{tabular}{|c|c|c|c|c|}
\hline Semestre & $\begin{array}{c}\text { Fase } \\
\text { formativa }\end{array}$ & Caracterización & Contexto/Nivel educativo & $\begin{array}{l}\text { Permanencia en el } \\
\text { centro de práctica }\end{array}$ \\
\hline I y II & Inicial & $\begin{array}{l}\text { Se insertan en diferentes espacios del sistema } \\
\text { escolar, para conocerlos y comenzar a inte- } \\
\text { ractuar en ellos. } \\
\text { Se incorporan al aula en duplas de trabajo. } \\
\text { Taller de reflexión semanal. }\end{array}$ & $\begin{array}{l}\text { - Jardines infantiles particu- } \\
\text { lares (niños } 0 \text { a } 6 \text { años). } \\
\text { - Escuelas municipales } \\
\text { (niños } 6 \text { a } 11 \text { años). } \\
\text { - Propio colegio del profesor } \\
\text { en formación. } \\
\text { - Escuelas especiales y de } \\
\text { lenguaje (preescolares, } \\
\text { niños y adultos). } \\
\text { - Proyectos integración } \\
\text { educativa en escuelas } \\
\text { municipales. }\end{array}$ & $\begin{array}{l}\text { Una mañana a la } \\
\text { semana. Práctica } \\
\text { mensual. }\end{array}$ \\
\hline III y IV & Intermedia & $\begin{array}{l}\text { Se insertan en unidades educativas que } \\
\text { trabajan con niños y niñas en situación de } \\
\text { vulnerabilidad social, lo que posibilita abrir } \\
\text { la experiencia formativa a un contexto edu- } \\
\text { cativo complejo, desafiante y diverso, en el } \\
\text { cual se espera que los(las) estudiantes pongan } \\
\text { en juego las representaciones explícitas e } \\
\text { implícitas sobre quién es el niño o niña y } \\
\text { cómo aprende. } \\
\text { Se incorporan al aula en duplas de trabajo. } \\
\text { Taller de reflexión semanal. }\end{array}$ & 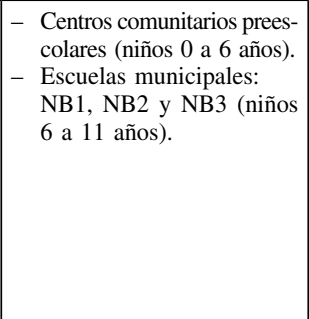 & $\begin{array}{l}\text { Una mañana a la } \\
\text { semana. Práctica } \\
\text { semestral. }\end{array}$ \\
\hline V y VI & Intermedia & $\begin{array}{l}\text { Se insertan en el ámbito específico en el que } \\
\text { tendrá lugar su futuro desempeño, con el pro- } \\
\text { pósito de que pueda diseñar, implementar y } \\
\text { evaluar proyectos específicos de intervención } \\
\text { didáctica en el nivel inicial con foco en niños } \\
\text { de } 0 \text { a } 3 \text { años, durante el primer semestre, y } \\
\text { de } 3 \text { a } 6 \text { años durante el segundo. La prác- } \\
\text { tica se desarrolla en unidades educativas de } \\
\text { dependencia particular o privada. } \\
\text { Se incorporan al aula de manera individual. } \\
\text { Taller de reflexión semanal }\end{array}$ & $\begin{array}{l}\text { - Niveles sala cuna menor y } \\
\text { mayor de jardines infanti- } \\
\text { les particulares (niños } 0 \text { a } \\
3 \text { años). } \\
\text { - Niveles medio, transi- } \\
\text { ción menor y mayor de } \\
\text { Colegios particulares } \\
\text { (niños } 3 \text { a } 6 \text { años). }\end{array}$ & $\begin{array}{l}\text { Una mañana a la } \\
\text { semana. Práctica } \\
\text { semestral. }\end{array}$ \\
\hline $\begin{array}{l}\text { VII y } \\
\text { VIII }\end{array}$ & Profesional & $\begin{array}{l}\text { Fase final del proceso de articulación teoría } \\
\text { y práctica desarrollado durante el período de } \\
\text { formación de los estudiantes, en la que se } \\
\text { les solicita diseñar, implementar y evaluar } \\
\text { proyectos específicos de intervención didác- } \\
\text { tica en el nivel inicial considerando, en un } \\
\text { primer momento (primer semestre), al niño } \\
\text { que aprende e incorporando en el segundo } \\
\text { semestre, a la familia y la comunidad a la } \\
\text { que pertenece. } \\
\text { Se incorporan al aula de manera individual. } \\
\text { Taller de reflexión semanal. }\end{array}$ & $\begin{array}{l}\text { - Escuelas municipales: } \\
\text { Niveles transición menor y } \\
\text { mayor (niños } 4 \text { a } 5 \text { años) } \\
\text { Se da continuidad a la } \\
\text { experiencia del primer } \\
\text { semestre en el mismo } \\
\text { grupo curso }\end{array}$ & $\begin{array}{l}\text { Una mañana a la } \\
\text { semana. Práctica } \\
\text { semestral. } \\
\text { Tres mañanas a la } \\
\text { semana. Práctica } \\
\text { semestral. }\end{array}$ \\
\hline
\end{tabular}




\section{METODOLOGIA}

El instrumento aplicado en la presente investigación proviene del proyecto FONDECYT ( No 1070807) "El Impacto de la Formación Práctica en el Proceso de Aprender a Enseñar", que ha estudiado el proceso de práctica progresiva de futuros docentes de 10 carreras de Pedagogía en Educación Básica y 11 carreras de Pedagogía Media en Ciencias (4), en Matemática (4) y en Lenguaje y Comunicación (3), pertenecientes a 10 Universidades chilenas ${ }^{2}$. El propósito de esta investigación es determinar las condiciones de la formación práctica que favorecen y obstaculizan el desarrollo de las competencias docentes asociadas al aprender a enseñar, a partir del análisis de las relaciones entre la oferta curricular del eje de práctica, las oportunidades de desempeño ofrecidas en los centros de práctica y las creencias de los profesores en formación. Dentro de este último aspecto, de sus creencias de autoeficacia.

Para cumplir con los objetivos de la presente investigación, se llevaron a cabo dos aplicaciones de la versión de 24 ítems -traducida al castellano- de la Escala de Sentido de Autoeficacia de los Profesores de Tschannen-Moran \& Woolfolk Hoy (2001) a alumnos de la carrera de Educación Parvularia de la UDP.

La decisión de realizar dos mediciones distintas se tomó con el fin de tener datos representativos de todos los semestres de la carrera, dado que en cada semestre los (las) estudiantes vivencian una experiencia distinta de contacto con el mundo escolar, la que se encuadra en el foco del taller de práctica correspondiente a ese semestre (cada semestre posee un propósito formativo distinto, que determina en qué espacio del sistema educativo ha de desarrollarse la experiencia). La primera medición se llevó a cabo en abril de 2008, a un grupo de 76 alumnos de los semestres I, III, V y VII. En la segunda medición participaron 62 alumnos, que en esa fecha cursaban los semestres II, IV, VI y VIII de la carrera (noviembre, 2008). De esta forma, se contó finalmente con 138 aplicaciones del instrumento.

La escala de autoeficacia utilizada contempla 24 ítems, los que se agrupan de acuerdo a tres dimensiones a medir: eficacia en el involucramiento de los estudiantes, eficacia de las estrategias de enseñanza y eficacia en el manejo de la clase. Para cada ítem el estudiante debe responder en base a una escala de Likert de 9 valores, donde el valor 1 representa que no se considera apto para llevar a cabo la acción propuesta en la pregunta (menor nivel de autoeficacia) y el valor 9 refleja que el estudiante se considera completamente capaz de lograr aquel objetivo (mayor nivel de autoeficacia).

\section{PARTICIPANTES}

Se invitó a participar a todas las estudiantes de la carrera de Educación Parvularia de la Facultad de Educación de la Universidad Diego Portales (en ese momento no cursaban la carrera estudiantes varones). La participación fue voluntaria y confidencial.

\footnotetext{
1 El equipo investigador es formado por Carmen Montecinos de la P. Universidad Católica de Valparaíso; Cristina Solís, Sylvia Rittershaussen, Inés Contreras, Claudio Núñez de la P. Universidad Católica de Chile y Horacio Walker, de la Universidad Diego Portales.

2 Universidad Andrés Bello, Universidad del Biobío, Universidad Católica de Chile, P. Universidad Católica de Valparaíso, Universidad Católica de Temuco, Universidad de Concepción, Universidad Diego Portales, Universidad Metropolitana de Ciencias de la Educación, Universidad de Playa Ancha, Universidad de la Santísima Trinidad de Concepción, .
} 
Las mediciones se llevaron a cabo en dos tiempos, donde participaron un grupo máximo de 76 estudiantes en la primera medición, grupo que disminuyó a 62 estudiantes para la última medición.

\section{PLAN DE ANALISIS}

Este estudio utilizó una escala de autoeficacia que originalmente se encontraba en idioma inglés, por lo que fue necesario evaluar la consistencia interna del instrumento -para nuestra población- con la prueba de alfa de Cronbach. Para esta prueba se utilizaron conjuntamente los datos de todas las aplicaciones, obteniéndose como resultado una consistencia interna alta (alfa $=0.94$ ), lo que se considera adecuado (Lévy \& Varela, 2003). Por otra parte, estos datos fueron también sometidos a un análisis factorial exploratorio, que evaluara el ajuste de éstos a las tres dimensiones (factores) de autoeficacia que mide originalmente el instrumento.

Este último análisis arrojó una medida de adecuación muestral de Kaiser-Meyer-Olkin de 0.92 y una prueba de esfericidad de Bartlett significativa $\left(x^{2}=1903.83, \mathrm{gl}=276\right.$, $p=0.00$ ), lo que muestra que la matriz de correlaciones de las respuestas a la prueba de autoeficacia es adecuada para la utilización de la técnica de análisis factorial.

A continuación, los datos se contrastaron con un modelo de tres factores (correspondientes a las tres dimensiones que evalúa el test de autoeficacia) con la técnica de componentes principales, resultando que, después de una rotación con Varimax, éstos explicaban el 58\% de la varianza conjunta de los ítems, haciéndolo de manera más o menos equitativa. Esta solución coincide con los tres factores que declara la escala utilizada, lo que confirma la posibilidad de uso, en esta muestra, del test ya traducido.

Habiendo despejado la duda de la adecuación del instrumento a la población evaluada, se diseñó el siguiente plan de análisis de datos para responder las preguntas planteadas en este estudio. Dado que esta investigación se dirige a conocer la manera en que evoluciona el sentido de autoeficacia de las alumnas a lo largo de su formación pedagógica, las variables dependientes utilizadas fueron los resultados globales en cada una de las tres dimensiones (factores) medidas por el test de autoeficacia: eficacia en el involucramiento de los estudiantes, eficacia de las estrategias de enseñanza y eficacia en el manejo de la clase; observando también, para algunos casos, las respuestas a ítems puntuales que ilustraran alguna tendencia específica.

De esta manera, en primer lugar se llevó a cabo un análisis descriptivo de las respuestas, para después realizar comparaciones de promedios de los puntajes en el test de autoeficacia. En estas comparaciones se utilizaron como variables independientes los semestres de la carrera (semestres I al VIII) que las alumnas cursaban al momento de tomar el test, dado que cada semestre representa la participación de las alumnas en una experiencia de práctica particular, conforme a la lógica de la malla curricular de la Carrera de Educación Parvularia. Con estos datos se llevó a cabo una comparación de los promedios de las tres dimensiones (factores), tomando en cuenta los semestres que los datos representaban. Estas comparaciones se llevaron a cabo con la prueba estadística de ANOVA, para luego llevar a efecto comparaciones particulares con el método de Bonferroni y con la prueba estadística $t$. Todos estos análisis se llevaron a cabo con el paquete estadístico SPSS 16.0. 


\section{RESULTADOS}

\section{ANALISIS DESCRIPTIVO}

Una exploración descriptiva de los patrones de respuesta de las alumnas muestra que, tomando en cuenta las respuestas al test como un todo, la respuesta de los sujetos es homogénea. En general, es una respuesta optimista, con valores mínimos de 4.96 y máximos de 9.0, y que alcanza un promedio de $\mathrm{M}=7.31(\mathrm{DS}=0.80)^{3}$.

Al mirar los resultados de acuerdo a cada factor, vemos que el factor eficacia en el involucramiento de los estudiantes (en adelante factor 1) promedia $\mathrm{M}=7.42$ (DS = 0.92), el factor eficacia de las estrategias de enseñanza (factor 2) alcanza una media de $\mathrm{M}=7.33$ (DS $=0.89)$ y el factor eficacia en el manejo de la clase (factor 3 ) tiene una media de $\mathrm{M}=7.07$ (DS = 0.91). Estas medias confirman tanto el optimismo de las alumnas como la homogeneidad de respuestas. No sólo son todos los promedios de un valor alto, sino que las respuestas de la muestra a los tres factores presentan tendencias similares, lo que se comprueba al observar la correlación positiva entre ellos (todas significativas a un nivel de $\mathrm{p}<0.001)$.

Aun cuando los promedios de los tres factores parecen similares, se observa una diferencia significativa entre el factor 3 (eficacia en el manejo de la clase) al compararlo, tanto con el factor $1(t=-5.30, \mathrm{gl}=133, p=0.00)$, como con el factor $2(t=3.96$, $\mathrm{gl}=135, p=0.00)$. En ambos casos, el promedio del factor 3 resulta significativamente más bajo.

En la tabla 2 podemos apreciar los promedios de los tres factores medidos, de acuerdo al semestres que cursaban las alumnas. Esta información también se puede observar en el gráfico 1 .

Tabla 2

Promedio en los tres factores de acuerdo a semestres en curso

\begin{tabular}{|c|c|c|c|}
\hline Semestre & Factor 1 & Factor 2 & Factor 3 \\
\hline I & 7.86 & 7.73 & 7.28 \\
\hline II & 7.23 & 6.94 & 7.11 \\
\hline III & 7.52 & 7.38 & 7.40 \\
\hline IV & 6.71 & 6.95 & 6.33 \\
\hline V & 7.46 & 7.02 & 6.95 \\
\hline VI & 7.68 & 7.62 & 6.43 \\
\hline VII & 7.04 & 7.25 & 6.78 \\
\hline VIII & 7.55 & 7.51 & 6.99 \\
\hline
\end{tabular}

3 Recordemos que las respuestas a los ítems se calificaban en base a una escala de Likert de 9 valores, donde un mayor valor corresponde a un mayor sentido de autoeficacia. 


\section{Gráfico 1}

Promedio por factor de acuerdo a semestre

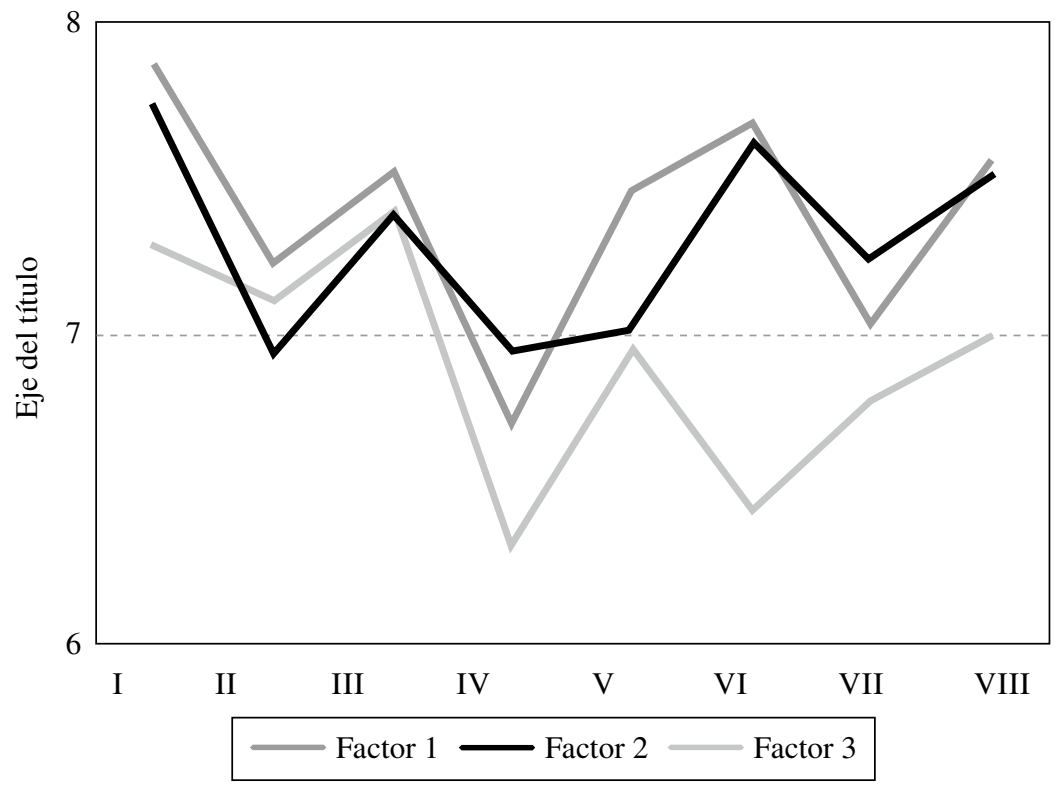

\section{ANALISIS INFERENCIAL: COMPARACIONES DE ACUERDO A SEMESTRE}

Como ya se ha mencionado, a cada semestre de la carrera de educación parvularia le corresponde una experiencia de práctica particular. Por esto, al comparar el nivel de autoeficacia reportado por las alumnas entre los diferentes semestres, estamos también observando la fluctuación de los puntajes asociados a las diferentes experiencias de práctica. Al realizar el ejercicio de comparar los promedios de cada factor de acuerdo al semestre que las alumnas cursan en el momento de tomar el test de autoeficacia, vemos que existen diferencias significativas para los factores 1 y 3 (factor $1, F=2.64, \mathrm{gl}=7$, $p=0.01$; factor $3, F=2.55, \mathrm{gl}=7, p=0.01$ ).

Después de llevar a cabo comparaciones particulares con el método de Bonferroni, se puede observar que las diferencias se encuentran, para el caso del factor 1, al comparar los promedios de los semestres I y IV $(p=0.01)$. En este caso particular, el promedio del factor 1 es significativamente menor en el semestre IV. O, en otras palabras, para el total de alumnas evaluadas el promedio en el factor de involucramiento de los estudiantes es significativamente mayor al comenzar sus estudios que en el semestre IV de la carrera de Educación Parvularia.

Respecto al factor 3, las diferencias se encuentran tanto al comparar los semestres III y IV, donde el promedio para el semestre IV es significativamente menor $(p=0.02)$. Asimismo, al comparar los semestres IV y VI también se observan diferencias significativas, donde el promedio del factor 3 en el semestre VI es significativamente mayor al del IV semestre $(p=0.03)$. En otras palabras, esto muestra que el factor del test de 
autoeficacia que se refiere a eficacia en el manejo de la clase disminuye significativamente desde el semestre III al IV de la carrera, para luego producirse un alza también significativa para el semestre VI.

Una manera de graficar estos cambios es observar el comportamiento de las preguntas más representativas para cada factor. Así, respecto del factor 1, eficacia en el involucramiento de los estudiantes, las siguientes preguntas reflejan de buena forma las variaciones:

1. ¿Cuánto puedes hacer para motivar a los estudiantes que muestran poco interés por el trabajo escolar? Las respuestas a esta pregunta promedian para el primer semestre $M=8.16$, lo que baja a $M=6.92$, en el IV semestre.

2. ¿Cuánto puedes hacer para que un estudiante con bajo rendimiento logre mejorar su comprensión del contenido? El promedio de las respuestas a esta pregunta presenta una media de $M=7.68$ en el primer semestre, la que disminuye a $M=6.50$, para el IV semestre.

3. ¿Cuánto puedes hacer para ayudar a los apoderados para que ellos apoyen el trabajo escolar de sus hijos? En el primer semestre, esta pregunta promedia $M=8.11$, mientras que en el IV, $M=5.25$, lo que refleja una baja importante.

Al estudiar el comportamiento de algunas preguntas que conforman el factor 3, eficacia en el manejo de la clase, se observa lo siguiente:

1. ¿Cuánto puedes hacer para controlar las conductas de los estudiantes que interrumpen el buen desarrollo de la clase? Efectivamente, el promedio de respuesta disminuye entre los semestres III y IV (de $M=7.38$ a $M=6.58$ ), para luego subir para el VI semestre $(M=7.18)$.

2. La pregunta ¿cuán bien puedes establecer rutinas de aula que permitan que las actividades de clase se desarrollen sin problemas? se comporta de una manera similar, disminuyendo su promedio entre los semestres III y IV (de $M=7.29$ a $M=6.50$ ), y volviendo a subir para el VI semestre $(M=7.59)$.

3. Esto también ocurre, de manera más acusada, para la pregunta ¿cuánto puedes hacer para lograr que los estudiantes respeten las normas y reglas del aula? Así, se observa que disminuye su nivel de autoeficacia entre los semestres III y IV (de $M=7.86$ a $M=6.33)$, para subir en el VI semestre $(M=7.71)$.

4. Finalmente, un comportamiento similar se observa para las preguntas ¿cuánto puedes hacer para controlar a un estudiante que tiene conductas que interrumpen el buen desarrollo de la clase?; y ¿cuán bien puedes evitar que unos pocos estudiantes "problemáticos" arruinen el desarrollo de una clase?, las que disminuyen en su nivel de autoeficacia entre los semestres II y IV (de $M=7.10$ a $M=6.08$, y de $M=7.38$ a $M=6.17$, respectivamente), para luego ambas aumentar en el semestre VI (a $M=7.0$ y $M=7.47$, respectivamente).

\section{DISCUSION}

Los resultados antes expuestos apoyan parcialmente la hipótesis del aumento del sentido de autoeficacia a medida que se avanza en la formación inicial y se cuenta con 
una mayor experiencia de práctica pedagógica. En efecto, los datos muestran que se presenta una fluctuación del sentido de eficacia entre los semestres y que no todos los factores asociados a la autoeficacia -medidos en esta investigación- se comportan de igual manera. Así, a lo largo de este apartado se intentará dar sentido a estos datos.

A la luz de lo que se ha venido señalando en este trabajo respecto del rol que juega el contexto como determinante en el desarrollo de aprendizajes durante la formación docente $y$, en particular, cómo esto es relevado en la propuesta de formación práctica específicamente estudiada, es posible establecer algunas vinculaciones entre los resultados reportados en cada momento del trayecto formativo (semestres) y los contextos de práctica, entendidos éstos en un sentido más amplio a partir de la caracterización realizada (lugar en que se desarrolla experiencia, foco de la práctica, entre otros).

En primer lugar, los resultados globales de cada factor mostraron que era el factor 3 el que significativamente presentaba el menor promedio de manera sostenida a lo largo de los ocho semestres de estudio. Esto podría explicarse a partir del choque de perspectivas que presumiblemente experimentan los estudiantes al imbuirse en las experiencias de práctica, independiente de a qué práctica nos estemos refiriendo. Esto ocurre porque, debido a su formación universitaria, los alumnos enfrentan las experiencias de práctica provistos de una serie de planteamientos y teorías actualizadas acerca de cómo gestionar una clase, que hoy en día se sustentan en la habilidad del docente para generar en los estudiantes la capacidad de autorregular su comportamiento, involucrándolos en la coconstrucción de normas que regulan las interacciones en el aula, siempre desde la perspectiva socioconstruida que predomina en el discurso pedagógico actual. Este enfoque se contradice profundamente con la realidad general de las aulas de clases de las escuelas. En efecto, tal como señalan Putnam y Borko, "los contextos disponibles para el trabajo de campo de los estudiantes de formación del profesorado no ejemplifican el tipo de enseñanza que los programas de formación del profesorado intentan promover" (2000, p. 4). Así, es muy posible suponer que una aproximación a la problemática del manejo de la clase y la disciplina desde cómo se entiende en las aulas reales, o sea, desde una perspectiva coercitiva y externa, en contraposición a la concepción recomendada durante su formación que está fundamentada en el desarrollo de la autorregulación de dichos procesos, puede generar en el profesor aprendiz la sensación de estar desprovisto de recursos y estrategias para manejar la clase adecuadamente.

Asimismo, desde otra perspectiva puede suponerse que apostar por el desarrollo de la autorregulación de los estudiantes implica por parte de éstos un proceso comprensivo y gradual, que supone una construcción colectiva del grupo, y que difícilmente puede rendir frutos en una intervención semanal o episódica, como es el contacto que sostienen los alumnos en práctica con los cursos. Este escenario probablemente ha de llevar al profesor en formación a cuestionarse la eficacia de dicho enfoque. De esta forma, ambos escenarios descritos pueden llevar a los practicantes a experimentar una sensación de ineficacia en el manejo de la clase.

Por otra parte, para intentar explicar las fluctuaciones observadas entre los semestres de estudio, para los factores medidos, se puede discutir lo siguiente: en relación al factor 1, eficacia en el involucramiento de los estudiantes, es posible señalar que el hecho de que los estudiantes se perciban a sí mismos respecto de este factor como menos eficaces al llegar al IV semestre de la carrera, puede responder, en primer lugar, al grado de distanciamiento que poseen de la realidad del aula en un primer momento. De hecho, 
es posible observar que desde un primer momento (primer semestre) los alumnos ya presentan un elevado sentido de autoeficacia en los tres factores evaluados. Y es posible que esta suerte de "ilusión de autoeficacia" vaya enfrentándose progresivamente con una realidad que la desmiente. En efecto, las experiencias de práctica que enfrentan los estudiantes durante el primer año (I y II semestres) corresponden a pasantías mensuales, en las que una vez por semana se incorporan a distintas realidades. De esta suerte de "vitrineo" por el sistema escolar, no se espera que asuman tareas específicas como motivar a los estudiantes, sino más bien que se aproximen al aula desarrollando distintas acciones puntuales, propias de la docencia. Dado que esto no corresponde a tareas por las que los profesores en formación deban responsabilizarse, ni conocer en profundidad en un primer momento, es probable que tengan la percepción de que éstas son fácilmente abordables y relativamente sencillas de desarrollar.

Este sentimiento inicial también se puede explicar por la mayor cercanía temporal, del profesor en formación, a su socialización primaria con el quehacer docente, que no es otra que su experiencia escolar como estudiante (Tardif, 2004). Claramente esta experiencia se encuentra más cercana en el tiempo que su rol futuro como docente formal. Así, es probablemente en este momento, en el inicio de la carrera, que -a diferencia de lo que ocurre en el IV semestre- desconoce las reales complejidades que encierra hacerse cargo de la enseñanza.

Por otra parte, el III semestre tampoco reviste mayor complejidad para los estudiantes dado que las prácticas tienen lugar en centros educativos de educación inicial. Así, por un lado, se vinculan con niños pequeños y, por otro, ese espacio (educación inicial) corresponde al contexto en el que ejercerán su labor profesional como futuras educadoras de párvulos. Este momento probablemente ha de resultarles más propicio en cuanto a posibilidades de desempeño, como también más cercano en términos de sus representaciones sobre el ejercicio de su futura profesión.

Sin embargo, en el IV semestre, que corresponde al último del plan común que la carrera de Pedagogía en Educación Parvularia comparte con la de Educación General Básica, las estudiantes se insertan en escuelas municipales en sectores vulnerables. De este modo, es probable que en este punto, tanto el hecho de que el nivel de enseñanza básica no corresponda al nivel en que se desempeñarán a futuro, como la alta complejidad que revisten las escuelas municipales (por lo general cursos muy numerosos, falta de normalización, procesos educativos interrumpidos, etc.), puedan afectar las expectativas acerca de su propio desempeño. Lo recién señalado podría explicar también por qué el factor 3, Eficacia en el manejo de la clase, decrece entre el I y IV semestres.

Por otra parte, si bien los resultados muestran cambios significativos asociados a los factores de autoeficacia 1 y 3, también muestran que el factor 2 (eficacia en las estrategias de enseñanza) se mantiene estable durante todos los semestres de estudio. Al respecto, es muy probable que la escasa fluctuación que presenta se explique por el foco en el que, tanto los estudiantes de pedagogía como los programas de formación inicial docente de nuestro país (no sólo en la propuesta estudiada), tienen puesto su énfasis. Este énfasis suele estar puesto en la enseñanza de los profesores, sin que ello necesariamente encuentre un correlato en los aprendizajes de los niños. Así, se observa en nuestra realidad una tendencia bastante generalizada a no considerar que lo que verdaderamente informa -respecto de una buena práctica de enseñanza- es el nivel de logro alcanzado por el(la) niño(a) que aprende. Este planteamiento, paradójico pero real, que da cuenta de una 
percepción episódica del proceso de enseñanza y aprendizaje por cuanto se asume sólo desde el polo del docente que enseña, desconoce que este es un proceso descentrado o multicentrado (Molina, 2000). De este modo, este sesgo se refleja presumiblemente en una valoración sostenidamente positiva de la eficacia en las estrategias de enseñanza, por cuanto estas no necesariamente son analizadas y/o reflexionadas, por parte de los futuros educadores de párvulos, a la luz de su eficiencia en los aprendizajes de los niños que forman parte de sus experiencias de práctica.

Otro argumento que podría explicar la estabilidad de este factor es el hecho de que en la reflexión que se intenta desarrollar en los talleres de práctica siempre prevalece una orientación crítica para comprender la realidad en la que se insertan. Así, esto implica que los estudiantes siempre están mirando el proceso educativo y lo que ocurre al interior del aula (como el desempeño del docente a cargo) desde una perspectiva crítica que, inevitablemente, les hace identificar y analizar el gran distanciamiento que hay entre el docente en ejercicio en esa aula y los planteamientos que ellos van construyendo sobre la enseñanza a partir de su formación profesional (anclada en una perspectiva crítica). Y esto lleva a que la valoración del desempeño del profesor en ejercicio con que se encuentran en el aula sea baja en relación a la percepción de su propio desempeño. De este modo, el tener estos profesores como parámetro hace que inevitablemente se perciban a sí mismos de manera más competente y con un mayor y mejor repertorio de estrategias de enseñanza.

Por otra parte, respecto de la progresiva recuperación de un sentido de autoeficacia positivo entre el IV y VI semestres, es posible señalar que desde el V semestre en adelante el plan de estudios se ha diferenciado, y las estudiantes ya se encuentran en la especialidad de Pedagogía en Educación Parvularia. Ello implica en términos formativos dos grandes cambios. Por un lado, que las experiencias de formación práctica que vivencian las estudiantes se desarrollan sólo en contextos educativos de educación inicial (centros de atención a niños de 0 a 6 años: jardines, salas cuna, "play groups", entre otros) y que los ramos teóricos que comienzan a cursar corresponden todos al ámbito específico de la educación inicial. Ambas situaciones, probablemente, inciden en una disposición personal y profesional a sentirse progresivamente más competentes, seguras y capaces de desarrollar las tareas propias de la enseñanza en el nivel inicial y, por ende, con un mayor sentido de autoeficacia docente. Asimismo, es probable que el trabajo desarrollado en el taller de reflexión sobre la experiencia que se lleva a cabo asociado a cada experiencia de práctica que vivencian las estudiantes, posibilite la toma de conciencia progresiva de las competencias profesionales que van adquiriendo y desarrollando conforme avanzan en su formación.

En resumen, estos resultados muestran que el sentido de autoeficacia tiende a fluctuar durante la formación pedagógica inicial, observándose especialmente desafiado cuando las alumnas se enfrentan a contextos educativos más complejos, como es el caso de los de dependencia municipal. En este punto, no hay que perder de vista que este estudio se realizó en el marco de la carrera de educación parvularia, donde se podría suponer que el contexto de educación básica (municipal, o no) se enfrenta como un desafío especialmente complejo, pues no es el lugar para el que se están preparando. Así, a futuro sería relevante llevar a cabo una medición de autoeficacia entre los alumnos que se forman, en la UDP, para ser profesores de educación básica, lo que permitiría confirmar las fluctuaciones y disminución de la autoeficacia al enfrentar las prácticas en contextos de dependencia municipal. 
Aún así, y dado que diversas investigaciones han mostrado de manera consistente una relación positiva entre una mayor autoeficacia de los docentes y el logro académico de los alumnos (Ross, 1992; Tschannen-Moran \& Woolfolk Hoy, 2001), la implementación de intervenciones dirigidas a aumentar el sentimiento de autoeficacia entre los profesores en formación puede resultar crucial para el esfuerzo de mejoramiento de la labor docente de ellos en el futuro. Así, aunque enfrentar contextos educativos difíciles sea algo que todo estudiante de pedagogía seguramente vivirá, no hay que olvidar que -de acuerdo a Bandura (1997) - los cambios en las creencias de autoeficacia no sólo se basan en el desempeño per se, sino que, principalmente, en el procesamiento cognitivo de la información diagnóstica que entrega el desempeño. Este significado que se le otorga a la experiencia es el que justamente se puede acompañar desde una experiencia de práctica profesional reflexiva, bien diseñada. En este sentido, los datos de la presente investigación se convierten en una información clave que permitirá evaluar el modelo de prácticas profesionales en la Facultad de Educación de la UDP, dando pie a intervenciones especialmente dirigidas a fomentar el aumento de la autoeficacia de los futuros profesores a medida que avanzan en sus estudios.

Si bien los resultados mencionados serán de gran utilidad dentro del contexto de la carrera estudiada, difícilmente podrán ser generalizados a contextos mayores, dado lo pequeño y específico de la muestra. Estudios futuros con muestras más representativas serán necesarios para generalizar resultados de este tipo.

\section{REFERENCIAS BIBLIOGRAFICAS}

Allinder, R.M. (1994). The relationship between efficacy and the instructional practices of special education teachers and consultants. Teacher Education and Special Education, 17, 86-95.

Armor, D. et al. (1976). Analysis for the school preferred reading programs in selected Los Angeles minority schools. Santa Mónica, CA: The Rand Corporation.

Ashton. P. \& Webb, R. (1986). Making a difference: Teacher's sense of efficacy and student achievement. New York: Longman.

Bandura, A. (1977). Self-efficacy: toward a unifying theory of behavioral change. Psychological Review, 84, 191-215.

Bandura, A. (1997). Self-efficacy: The exercise of control. New York: Freeman.

Brown, J., Collins, A. \& Duguid, P. (1989). Situated Cognition and the Culture of Learning. Educational Researcher, 18, 32-42.

Bruner, J. (1997). La educación, puerta de la cultura. Madrid: Visor.

Bullough, R. (2000). Teacher education reform as a story of possibility: lessons learned, lessons forgotten-the American Council on Education's Commission on Teacher Education (1939-1942). Teaching and Teacher Education, 16, 131-145.

Caprara, G., Barbaranelli, C., Steca, P. \& Malone, P. (2006). Teacher's self-efficacy beliefs as determinants of job satisfaction and student's academic achievement: A study at the school level. Journal of School Psychology, 44, 473-490.

Carroll, A., Forlin, C. \& Jobling, A. (2003). The Impact of Teacher Training in Special Education on the Attitudes of Australian Preservice General Educators towards People with Disabilities. Teacher Education Quarterly, 65-79.

Clift, R. \& Brady, P. (2005). Research on methods courses and field experiencies. In CochranSmith, M. \& Zeichner, K.M. (Eds.), Studying teacher education: The report of the AERA 
panel on research and teacher education (pp. 209-424). Mahwah, NJ: Lawrence Erlbaum Associates Publishers.

Conway, P. \& Clark, C. (2003). The journey inward and outward: a re-examination of Fuller's concerns-based model of teacher development. Teaching and Teacher Education, 19, 465-482.

Del Valle, R., Ackley, B., Tagle, T. \& Flores, L. (2009). The Perceived Self-Efficacy Beliefs of Pre-Service Teachers of English. Annual Meeting of the American Educational Research Association. San Diego, California.

Díaz, F. y Hernández, G. (1998). Estrategias Docentes para un Aprendizaje Significativo. Una interpretación constructivista. Mc-Graw Hill Editores.

Fuller, F. \& Brown, O. (1975). Becoming a Teacher. In Ryan, K. (Ed.), Seventy-fourth year book of the National Society for the Study of Education: Pt. 2. Teacher Education. Chicago: University of Chicago Press.

Glickman, C. \& Tamashiro, R. (1982). A comparison of first-year, fifth-year, and former teachers on efficacy, ego development, and problem solving. Psychology in the Schools, 19, 558-562.

Goddard, R., Hoy, W. \& Woolfolk Hoy, A. (2004). Collective Efficacy Beliefs: Theoretical Developments, Empirical Evidence, and Future Directions Educational Researcher, 3, 3-13.

Gould, H. (2004). Can Novice Teachers Differentiate Instruction? Yes, They can! New Horizons for Learning.

Klassen, R., Bong, M., Usher, E., Chomg, W., Huan, V., Wong, I. \& Georgius, T. (2009). Exploring the validity of teacher's self-efficacy scale in five countries. Contemporary Educational Psychology, 34, 67-76.

Lévy, J.-P. \& Varela, J. (2003) Análisis Multivariable para las Ciencias Sociales. Editorial Pearson Educación, S.A. España.

Milner, R. (2002). Affective and Social Issues among High Achieving African American Students: Recommendations for Teachers and Teacher Education. Action in Teacher Education, 24, 81-89.

Molina, V. (2000). La actividad que permite el aprendizaje es acción acompañada de pensamiento. Docencia, 12, 35-46.

Pigge, F. \& Marso, R. (1990). A Longitudinal Assessment of the Affective Impact of Preservice Training on Prospective Teachers. Journal of Experimental Education, 58.

Putnam, R. \& Borko, H. (2000). What new ways of knowledge and thinking have to say about research on teacher learning. Educational Researcher, 29, 4-15.

Richards, G. \& Clough, P. (2004). ITE students' attitudes to inclusion. Research in Education, $72,77-86$.

Ross, J. (1992). Teacher efficacy and the effect of coaching on student achievement. Canadian Journal of Education, 17, 51-65.

Shaughnessy, M. (2004). An Interview with Anita Woolfolk: The Educational Psychology of Teacher Efficacy. Educational Psychology Review, 16, 153-176.

Shulman, L. (2005). Conocimiento y enseñanza: Fundamentos de la nueva reforma. Profesorado. Revista de currículum y formación del profesorado, 9, 2, 1-30.

Skaalvik, E. \& Skaalvik, S. (2007). Dimensions of teacher self-efficacy and relations with strain factors, perceived collective teacher efficacy, and teacher burnout. Journal of Educational Psychology, 99, 611-625.

Stein, M. \& Wang, M. (1988). Teacher development and school improvement: the process of teacher change. Teacher and Teaching education, 4, 171-187.

Tardif, N. (2004). Los saberes del docente y su desarrollo profesional. Editorial Narcea. Madrid, 2004. Pág. 15.

Tournaki, N. \& Podell, D. (2005). The impact of student characteristics and teacher efficacy on teachers' predictions of student success. Teaching and Teacher Education, 21, 299-314. 
Tschannen-Moran, M. \& Woolfolk Hoy, A. (2001). Teacher efficacy: Capturing an elusive construct. Teaching and Teacher Education, 17, 783-805.

Tschannen-Moran, M., Woolfolk Hoy, A. \& Hoy, W. (1998). Teacher efficacy: Its meaning and measure. Review of Educational Research, 68, 202-248.

Wolters, C. \& Daugherty, S. (2007). Goal Structures and Teachers' Sense of Efficacy: Their Relation and Association to Teaching Experience and Academic Level. Journal of Educational Psychology, 99, 181-193.

Woolfolk Hoy, A. \& Burke Spero, R. (2005). Changes in teacher efficacy during the early years of teaching: A comparison of four measures. Teaching and Teacher Education, 21, 343-356. 